\title{
The Sylow Theorems
}

\author{
Marco Riccardi \\ Casella Postale 49 \\ 54038 Montignoso, Italy
}

\begin{abstract}
Summary. The goal of this article is to formalize the Sylow theorems closely following the book [4]. Accordingly, the article introduces the group operating on a set, the stabilizer, the orbits, the $p$-groups and the Sylow subgroups.
\end{abstract}

MML identifier: GROUP_10, version: 7.8.05 4.87.985

The papers [20], [26], [18], [9], [21], [14], [11], [27], [6], [28], [7], [3], [5], [10], [1], [23], [24], [22], [16], [13], [19], [17], [2], [25], [15], [8], and [12] provide the notation and terminology for this paper.

\section{Group Operating on a Set}

Let $S$ be a non empty 1-sorted structure, let $E$ be a set, let $A$ be an action of the carrier of $S$ on $E$, and let $s$ be an element of $S$. We introduce $A^{\frown} s$ as a synonym of $A(s)$.

Let $S$ be a non empty 1-sorted structure, let $E$ be a set, let $A$ be an action of the carrier of $S$ on $E$, and let $s$ be an element of $S$. Then $A^{\frown} s$ is a function from $E$ into $E$.

Let $S$ be a unital non empty groupoid, let $E$ be a set, and let $A$ be an action of the carrier of $S$ on $E$. We say that $A$ is left-operation if and only if:

(Def. 1) $A^{\frown}\left(\mathbf{1}_{S}\right)=\operatorname{id}_{E}$ and for all elements $s_{1}, s_{2}$ of $S$ holds $A^{\frown}\left(s_{1} \cdot s_{2}\right)=$ $\left(A \frown s_{1}\right) \cdot\left(A \frown s_{2}\right)$.

Let $S$ be a unital non empty groupoid and let $E$ be a set. Note that there exists an action of the carrier of $S$ on $E$ which is left-operation.

Let $S$ be a unital non empty groupoid and let $E$ be a set. A left operation of $S$ on $E$ is a left-operation action of the carrier of $S$ on $E$. 
The scheme ExLeftOperation deals with a set $\mathcal{A}$, a group-like non empty groupoid $\mathcal{B}$, and a unary functor $\mathcal{F}$ yielding a function from $\mathcal{A}$ into $\mathcal{A}$, and states that:

There exists a left operation $T$ of $\mathcal{B}$ on $\mathcal{A}$ such that for every element $s$ of $\mathcal{B}$ holds $T(s)=\mathcal{F}(s)$

provided the parameters meet the following requirements:

- $\mathcal{F}\left(\mathbf{1}_{\mathcal{B}}\right)=\mathrm{id}_{\mathcal{A}}$, and

- For all elements $s_{1}, s_{2}$ of $\mathcal{B}$ holds $\mathcal{F}\left(s_{1} \cdot s_{2}\right)=\mathcal{F}\left(s_{1}\right) \cdot \mathcal{F}\left(s_{2}\right)$.

Next we state the proposition

(1) Let $E$ be a non empty set, $S$ be a group-like non empty groupoid, $s$ be an element of $S$, and $L_{1}$ be a left operation of $S$ on $E$. Then $L_{1} \frown s$ is one-to-one.

Let $S$ be a non empty groupoid and let $s$ be an element of $S$. We introduce $\gamma_{s}$ as a synonym of $s^{*}$.

Let $S$ be a group-like associative non empty groupoid. The functor $\boldsymbol{\Gamma}_{S}$ yielding a left operation of $S$ on the carrier of $S$ is defined as follows:

(Def. 2) For every element $s$ of $S$ holds $\boldsymbol{\Gamma}_{S}(s)=\gamma_{s}$.

Let $E$ be a set and let $n$ be a set. The functor $[E]^{n}$ yielding a family of subsets of $E$ is defined by:

(Def. 3) $[E]^{n}=\{X ; X$ ranges over subsets of $E: \overline{\bar{X}}=n\}$.

Let $E$ be a finite set and let $n$ be a set. One can verify that $[E]^{n}$ is finite.

The following two propositions are true:

(2) For every natural number $n$ and for every non empty set $E$ such that $\overline{\bar{n}} \leq \overline{\bar{E}}$ holds $[E]^{n}$ is non empty.

(3) For every non empty finite set $E$ and for every element $k$ of $\mathbb{N}$ and for all sets $x_{1}, x_{2}$ such that $x_{1} \neq x_{2}$ holds card $\operatorname{Choose}\left(E, k, x_{1}, x_{2}\right)=\operatorname{card}\left([E]^{k}\right)$.

Let $E$ be a non empty set, let $n$ be a natural number, let $S$ be a group-like non empty groupoid, let $s$ be an element of $S$, and let $L_{1}$ be a left operation of $S$ on $E$. Let us assume that $\overline{\bar{n}} \leq \overline{\bar{E}}$. The functor $\gamma_{s, L_{1}}^{n}$ yields a function from $[E]^{n}$ into $[E]^{n}$ and is defined by:

(Def. 4) For every element $X$ of $[E]^{n}$ holds $\gamma_{s, L_{1}}^{n}(X)=\left(L_{1} \bumpeq s\right)^{\circ} X$.

Let $E$ be a non empty set, let $n$ be a natural number, let $S$ be a group-like non empty groupoid, and let $L_{1}$ be a left operation of $S$ on $E$. Let us assume that $\overline{\bar{n}} \leq \overline{\bar{E}}$. The functor $\Gamma_{L_{1}}^{n}$ yields a left operation of $S$ on $[E]^{n}$ and is defined by:

(Def. 5) For every element $s$ of $S$ holds $\Gamma_{L_{1}}^{n}(s)=\gamma_{s, L_{1}}^{n}$.

Let $S$ be a non empty groupoid, let $s$ be an element of $S$, and let $Z$ be a non empty set. The functor $\gamma_{s, Z}$ yielding a function from : the carrier of $S, Z$ : into : the carrier of $S, Z$ :] is defined by the condition (Def. 6). 
(Def. 6) Let $z_{1}$ be an element of : the carrier of $S, Z$ :]. Then there exists an element $z_{2}$ of : the carrier of $S, Z$; and there exist elements $s_{1}, s_{2}$ of $S$ and there exists an element $z$ of $Z$ such that $z_{2}=\gamma_{s, Z}\left(z_{1}\right)$ and $s_{2}=s \cdot s_{1}$ and $z_{1}=\left\langle s_{1}, z\right\rangle$ and $z_{2}=\left\langle s_{2}, z\right\rangle$.

Let $S$ be a group-like associative non empty groupoid and let $Z$ be a non empty set. The functor $\Gamma_{S, Z}$ yields a left operation of $S$ on : the carrier of $S$, $Z$ : and is defined by:

(Def. 7) For every element $s$ of $S$ holds $\boldsymbol{\Gamma}_{S, Z}(s)=\gamma_{s, Z}$.

Let $G$ be a group, let $H, P$ be subgroups of $G$, and let $h$ be an element of $H$. The functor $\gamma_{h, P}$ yields a function from the left cosets of $P$ into the left cosets of $P$ and is defined by the condition (Def. 8).

(Def. 8) Let $P_{1}$ be an element of the left cosets of $P$. Then there exists an element $P_{2}$ of the left cosets of $P$ and there exist subsets $A_{1}, A_{2}$ of $G$ and there exists an element $g$ of $G$ such that $P_{2}=\gamma_{h, P}\left(P_{1}\right)$ and $A_{2}=g \cdot A_{1}$ and $A_{1}=P_{1}$ and $A_{2}=P_{2}$ and $g=h$.

Let $G$ be a group and let $H, P$ be subgroups of $G$. The functor $\boldsymbol{\Gamma}_{H, P}$ yields a left operation of $H$ on the left cosets of $P$ and is defined as follows:

(Def. 9) For every element $h$ of $H$ holds $\boldsymbol{\Gamma}_{H, P}(h)=\gamma_{h, P}$.

\section{Stabilizer AND Orbits}

Let $G$ be a group, let $E$ be a non empty set, let $T$ be a left operation of $G$ on $E$, and let $A$ be a subset of $E$. The functor $T_{A}$ yields a strict subgroup of $G$ and is defined as follows:

(Def. 10) The carrier of $T_{A}=\left\{g ; g\right.$ ranges over elements of $G$ : $\left.\left(T^{\frown} g\right)^{\circ} A=A\right\}$.

Let $G$ be a group, let $E$ be a non empty set, let $T$ be a left operation of $G$ on $E$, and let $x$ be an element of $E$. The functor $T_{x}$ yielding a strict subgroup of $G$ is defined by:

(Def. 11) $T_{x}=T_{\{x\}}$.

Let $S$ be a unital non empty groupoid, let $E$ be a set, let $T$ be a left operation of $S$ on $E$, and let $x$ be an element of $E$. We say that $x$ is fixed under $T$ if and only if:

(Def. 12) For every element $s$ of $S$ holds $x=\left(T^{\frown} s\right)(x)$.

Let $S$ be a unital non empty groupoid, let $E$ be a set, and let $T$ be a left operation of $S$ on $E$. The functor $T_{0}$ yields a subset of $E$ and is defined by:

(Def. 13) $T_{0}=\left\{\begin{aligned}\{x ; x \text { ranges over elements of } E: x \text { is fixed under } T\}, & \text { if } E \text { is non empty, } \\ \emptyset_{E}, & \text { otherwise. }\end{aligned}\right.$ 
Let $S$ be a unital non empty groupoid, let $E$ be a set, let $T$ be a left operation of $S$ on $E$, and let $x, y$ be elements of $E$. We say that $x$ and $y$ are conjugated under $T$ if and only if:

(Def. 14) There exists an element $s$ of $S$ such that $y=\left(T^{\frown} s\right)(x)$.

We now state three propositions:

(4) Let $S$ be a unital non empty groupoid, $E$ be a non empty set, $x$ be an element of $E$, and $T$ be a left operation of $S$ on $E$. Then $x$ and $x$ are conjugated under $T$.

(5) Let $G$ be a group, $E$ be a non empty set, $x, y$ be elements of $E$, and $T$ be a left operation of $G$ on $E$. Suppose $x$ and $y$ are conjugated under $T$. Then $y$ and $x$ are conjugated under $T$.

(6) Let $S$ be a unital non empty groupoid, $E$ be a non empty set, $x, y, z$ be elements of $E$, and $T$ be a left operation of $S$ on $E$. Suppose $x$ and $y$ are conjugated under $T$ and $y$ and $z$ are conjugated under $T$. Then $x$ and $z$ are conjugated under $T$.

Let $S$ be a unital non empty groupoid, let $E$ be a non empty set, let $T$ be a left operation of $S$ on $E$, and let $x$ be an element of $E$. The functor $T(x)$ yields a subset of $E$ and is defined as follows:

(Def. 15) $T(x)=\{y ; y$ ranges over elements of $E: x$ and $y$ are conjugated under $T\}$.

One can prove the following four propositions:

(7) Let $S$ be a unital non empty groupoid, $E$ be a non empty set, $x$ be an element of $E$, and $T$ be a left operation of $S$ on $E$. Then $T(x)$ is non empty.

(8) Let $G$ be a group, $E$ be a non empty set, $x, y$ be elements of $E$, and $T$ be a left operation of $G$ on $E$. Then $T(x)$ misses $T(y)$ or $T(x)=T(y)$.

(9) Let $S$ be a unital non empty groupoid, $E$ be a non empty finite set, $x$ be an element of $E$, and $T$ be a left operation of $S$ on $E$. If $x$ is fixed under $T$, then $T(x)=\{x\}$.

(10) Let $G$ be a group, $E$ be a non empty set, $a$ be an element of $E$, and $T$ be a left operation of $G$ on $E$. Then $\overline{\overline{T(a)}}=\left|\bullet: T_{a}\right|$.

Let $G$ be a group, let $E$ be a non empty set, and let $T$ be a left operation of $G$ on $E$. The orbits of $T$ yields a partition of $E$ and is defined by:

(Def. 16) The orbits of $T=\left\{X ; X\right.$ ranges over subsets of $E: \bigvee_{x \text { : element of } E} X=$ $T(x)\}$.

\section{3. $p$-GROUPS}

Let $p$ be a prime natural number and let $G$ be a group. We say that $G$ is a $p$-group if and only if: 
(Def. 17) There exists a natural number $r$ such that $\operatorname{ord}(G)=p^{r}$.

Let $p$ be a prime natural number, let $G$ be a group, and let $P$ be a subgroup of $G$. We say that $P$ is a $p$-group if and only if:

(Def. 18) There exists a finite group $H$ such that $P=H$ and $H$ is a $p$-group.

One can prove the following proposition

(11) Let $E$ be a non empty finite set, $G$ be a finite group, $p$ be a prime natural number, and $T$ be a left operation of $G$ on $E$. If $G$ is a $p$-group, then $\operatorname{card} T_{0} \bmod p=\operatorname{card} E \bmod p$.

\section{The Sylow Theorems}

Let $p$ be a prime natural number, let $G$ be a group, and let $P$ be a subgroup of $G$. We say that $P$ is a Sylow $p$-subgroup if and only if:

(Def. 19) $P$ is a $p$-group and $p \nmid|\bullet: P|_{\mathbb{N}}$.

We now state three propositions:

(12) For every finite group $G$ and for every prime natural number $p$ holds there exists a subgroup of $G$ which is a Sylow $p$-subgroup.

(13) Let $G$ be a finite group and $p$ be a prime natural number. If $p \mid \operatorname{ord}(G)$, then there exists an element $g$ of $G$ such that $\operatorname{ord}(g)=p$.

(14) Let $G$ be a finite group and $p$ be a prime natural number. Then

(i) for every subgroup $H$ of $G$ such that $H$ is a $p$-group there exists a subgroup $P$ of $G$ such that $P$ is a Sylow $p$-subgroup and $H$ is a subgroup of $P$, and

(ii) for all subgroups $P_{1}, P_{2}$ of $G$ such that $P_{1}$ is a Sylow $p$-subgroup and $P_{2}$ is a Sylow $p$-subgroup holds $P_{1}$ and $P_{2}$ are conjugated.

Let $G$ be a group and let $p$ be a prime natural number. The functor $\operatorname{Syl}_{p}(G)$ yielding a subset of $\operatorname{SubGr} G$ is defined as follows:

(Def. 20) $\operatorname{Syl}_{p}(G)=\{H ; H$ ranges over elements of $\operatorname{SubGr} G$ :

$\bigvee_{P \text { : strict subgroup of } G}(P=H \wedge P$ is a Sylow $p$-subgroup $\left.)\right\}$.

Let $G$ be a finite group and let $p$ be a prime natural number. Note that $\operatorname{Syl}_{p}(G)$ is non empty and finite.

Let $G$ be a finite group, let $p$ be a prime natural number, let $H$ be a subgroup of $G$, and let $h$ be an element of $H$. The functor $\gamma_{h, p}$ yielding a function from $\mathrm{Syl}_{p}(G)$ into $\mathrm{Syl}_{p}(G)$ is defined by the condition (Def. 21 ).

(Def. 21) Let $P_{1}$ be an element of $\operatorname{Syl}_{p}(G)$. Then there exists an element $P_{2}$ of $\mathrm{Syl}_{p}(G)$ and there exist strict subgroups $H_{1}, H_{2}$ of $G$ and there exists an element $g$ of $G$ such that $P_{2}=\gamma_{h, p}\left(P_{1}\right)$ and $P_{1}=H_{1}$ and $P_{2}=H_{2}$ and $h^{-1}=g$ and $H_{2}=H_{1}{ }^{g}$. 
Let $G$ be a finite group, let $p$ be a prime natural number, and let $H$ be a subgroup of $G$. The functor $\boldsymbol{\Gamma}_{H, p}$ yields a left operation of $H$ on $\operatorname{Syl}_{p}(G)$ and is defined as follows:

(Def. 22) For every element $h$ of $H$ holds $\boldsymbol{\Gamma}_{H, p}(h)=\gamma_{h, p}$.

The following proposition is true

(15) For every finite group $G$ and for every prime natural number $p$ holds $\operatorname{card}\left(\operatorname{Syl}_{p}(G)\right) \bmod p=1$ and $\operatorname{card}\left(\operatorname{Syl}_{p}(G)\right) \mid \operatorname{ord}(G)$.

\section{APPENDIX}

The following propositions are true:

(16) For all non empty sets $X, Y$ holds $\overline{\overline{\{: X,\{y\}:: y \text { ranges over elements of } Y\}}}=\overline{\bar{Y}}$.

(17) For all natural numbers $n, m, r$ and for every prime natural number $p$ such that $n=p^{r} \cdot m$ and $p \nmid m$ holds $\left(\begin{array}{c}n \\ p^{r}\end{array}\right) \bmod p \neq 0$.

(18) For every natural number $n$ such that $n>0$ holds ord $\left(\mathbb{Z}_{n}^{+}\right)=n$.

(19) For every group $G$ and for every non empty subset $A$ of $G$ and for every element $g$ of $G$ holds $\overline{\bar{A}}=\overline{\overline{A \cdot g}}$.

\section{REFERENCES}

[1] Grzegorz Bancerek. Cardinal numbers. Formalized Mathematics, 1(2):377-382, 1990.

[2] Grzegorz Bancerek. The ordinal numbers. Formalized Mathematics, 1(1):91-96, 1990.

[3] Grzegorz Bancerek and Krzysztof Hryniewiecki. Segments of natural numbers and finite sequences. Formalized Mathematics, 1(1):107-114, 1990.

[4] Nicolas Bourbaki. Elements of Mathematics. Algebra I. Chapters 1-3. Springer-Verlag, Berlin, Heidelberg, New York, London, Paris, Tokyo, 1989.

[5] Czesław Byliński. Finite sequences and tuples of elements of a non-empty sets. Formalized Mathematics, 1(3):529-536, 1990.

[6] Czesław Byliński. Functions and their basic properties. Formalized Mathematics, 1(1):5565, 1990.

[7] Czesław Byliński. Functions from a set to a set. Formalized Mathematics, 1(1):153-164, 1990.

[8] Czesław Byliński. Partial functions. Formalized Mathematics, 1(2):357-367, 1990.

[9] Czesław Byliński. Some basic properties of sets. Formalized Mathematics, 1(1):47-53, 1990.

[10] Czesław Byliński. The sum and product of finite sequences of real numbers. Formalized Mathematics, 1(4):661-668, 1990.

[11] Agata Darmochwal. Finite sets. Formalized Mathematics, 1(1):165-167, 1990.

[12] Artur Korniłowicz. The definition and basic properties of topological groups. Formalized Mathematics, 7(2):217-225, 1998.

[13] Rafał Kwiatek. Factorial and Newton coefficients. Formalized Mathematics, 1(5):887-890, 1990.

[14] Rafał Kwiatek and Grzegorz Zwara. The divisibility of integers and integer relative primes. Formalized Mathematics, 1(5):829-832, 1990.

[15] Karol Pąk. Cardinal numbers and finite sets. Formalized Mathematics, 13(3):399-406, 2005.

[16] Konrad Raczkowski and Paweł Sadowski. Equivalence relations and classes of abstraction. Formalized Mathematics, 1(3):441-444, 1990. 
[17] Dariusz Surowik. Cyclic groups and some of their properties - part I. Formalized Mathematics, 2(5):623-627, 1991.

[18] Andrzej Trybulec. Subsets of complex numbers. To appear in Formalized Mathematics.

[19] Andrzej Trybulec. Domains and their Cartesian products. Formalized Mathematics, 1(1):115-122, 1990.

[20] Andrzej Trybulec. Tarski Grothendieck set theory. Formalized Mathematics, 1(1):9-11, 1990.

[21] Michał J. Trybulec. Integers. Formalized Mathematics, 1(3):501-505, 1990.

[22] Wojciech A. Trybulec. Classes of conjugation. Normal subgroups. Formalized Mathematics, 1(5):955-962, 1990.

[23] Wojciech A. Trybulec. Groups. Formalized Mathematics, 1(5):821-827, 1990.

[24] Wojciech A. Trybulec. Subgroup and cosets of subgroups. Formalized Mathematics, $1(5): 855-864,1990$.

[25] Wojciech A. Trybulec and Michał J. Trybulec. Homomorphisms and isomorphisms of groups. Quotient group. Formalized Mathematics, 2(4):573-578, 1991.

[26] Zinaida Trybulec. Properties of subsets. Formalized Mathematics, 1(1):67-71, 1990.

[27] Edmund Woronowicz. Relations and their basic properties. Formalized Mathematics, 1(1):73-83, 1990.

[28] Edmund Woronowicz. Relations defined on sets. Formalized Mathematics, 1(1):181-186, 1990.

Received August 13, 2007 